\title{
Adipocinas: uma visão geral dos seus efeitos metabólicos
}

\author{
Adipokines: an overview of its metabolic effects
}

\author{
Amélio F. Godoy-Matos", Ivan C. Cruz, Rafael da Costa, Wellington S. Silva Júnior, \\ Brazilian Adipokines Study Group ${ }^{* *}$
}

\begin{abstract}
Resumo
Há um número crescente de evidências indicando que o tecido adiposo é um órgão endócrino capaz de sintetizar e secretar substâncias envolvidas na fisiopatogênese da resistência à insulina, da inflamação sistêmica e da aterogênese. O objetivo deste artigo é realizar uma revisão sobre as adipocinas, substâncias oriundas do tecido adiposo que parecem constituir o elo entre obesidade e doenças metabólicas e cardiovasculares. As adipocinas manifestam seus efeitos através de mecanismos endócrinos e parácrinos. Elas atuam como imunomoduladores, alterando o equilíbrio entre citocinas pró e anti-inflamatórias; influenciam a sensibilidade à insulina, interferindo na homeostase glicêmica; regulam o mecanismo central da fome e do gasto energético, com consequências diretas sobre o peso corporal. Além disso, apresentam efeitos sobre o tônus vascular, a proliferação celular e a aterogênese, modificando o risco cardiovascular, e influenciam outros fatores determinantes da síndrome metabólica, como os níveis pressóricos, o perfil lipídico e o grau de esteatose e inflamação hepáticas. As adipocinas podem ser ferramentas interessantes para uso clínico. Elas funcionam como biomarcadores de distúrbios metabólicos e ateroscleróticos, possibilitando a identificação precoce de indivíduos de risco cardiovascular aumentado. Além disso, a manipulação dessas substâncias apresenta um potencial terapêutico que não deve ser negligenciado, como já consagrado pela utilização dos inibidores da dipeptidil peptidase 4 para o tratamento do diabetes ou da leptina recombinante para pacientes com deficiência genética dessa adipocina. O uso desta última em pacientes lipodistróficos, bem como a administração de visfatina no momento da reperfusão do miocárdio, com benefícios já comprovados em modelos experimentais, também parecem ser promissores.
\end{abstract}

Descritores: Adipocinas; Obesidade; Síndrome metabólica; Resistência à insulina.

\begin{abstract}
There is a growing body of evidence indicating that adipose tissue is an endocrine organ able to synthesize and secrete substances involved in the pathogenesis of insulin resistance, systemic inflammation and atherogenesis. The purpose of this article is to conduct a review of the adipokines, adipose tissue-derived substances that seem to be the link between obesity and metabolic and cardiovascular diseases. Adipokines manifest their effects through endocrine and paracrine mechanisms. They act as immunomodulators, altering the balance between pro and anti-inflammatory cytokines; influence insulin sensitivity, interfering with glycemic homeostasis; regulate the central mechanism of appetite control and energy expenditure, with direct consequences on body weight. Moreover, they have effects on vascular tone, cell proliferation and atherogenesis, which modify cardiovascular risk, and they influence other determinants of the metabolic syndrome, such as blood pressure, lipid profile and degree of hepatic steatosis and inflammation. Adipokines may be an interesting tool for clinical use. They serve as biomarkers of metabolic and atherosclerotic disorders, enabling early identification of individuals at increased cardiovascular risk. In addition, handling these substances has a therapeutic potential that should not be overlooked, as suggested by the use of inhibitors of dipeptidyl peptidase 4 for the treatment of diabetes or by human recombinant leptin in patients with genetic deficiency of this adipokine. The use of the latter in lipodystrophic patients, as well as administration of visfatin for myocardial reperfusion with proven benefits in experimental models, also appears to be promising.
\end{abstract}

Keywords: Adipokines; Obesity; Metabolic syndrome; Insulin resistance. 


\section{Introdução}

A obesidade é um problema de saúde pública mundial. ${ }^{1}$ Em especial, a obesidade abdominal está associada com doenças metabólicas e cardiovasculares, o que a torna motivo de abundantes pesquisas na tentativa de esclarecer a magnitude do risco e os mecanismos associados com a obesidade. Um mecanismo plausível assenta-se no tecido adiposo como órgão endócrino, capaz de sintetizar e secretar substâncias envolvidas na fisiopatogênese da resistência à insulina (RI), inflamação sistêmica e aterogênese. ${ }^{2}$ A estas substâncias convencionou-se denominar "adipocinas". ${ }^{3}$ Elas são provenientes dos adipócitos, dos macrófagos e das células não adiposas da matriz. Além disso, embora a adiponectina e a leptina sejam liberadas no sangue como hormônios, outras adipocinas parecem ser fatores parácrinos cuja liberação pelo tecido adiposo pode não contribuir significativamente para os seus níveis circulantes. ${ }^{4} \mathrm{O}$ objetivo deste artigo é discutir brevemente algumas dessas adipocinas no contexto da obesidade e seu cortejo metabólico.

\section{Adiponectina}

Descoberta em $1995,{ }^{5}$ a adiponectina é uma adipocina de estrutura proteica cuja transcrição é regulada principalmente pelo peroxisome proliferator-activated receptor gamma (PPAR-gama). ${ }^{6}$ Sua síntese ocorre predominantemente no tecido adiposo, sendo a forma oligomérica, de alto peso molecular, a mais abundante. ${ }^{7}$ Atua através de três receptores transmembrana, AdipoR1, AdipoR2 e $T$-cadherin. Os dois primeiros predominam no tecido muscular esquelético e hepático, respectivamente. O papel fisiológico da adiponectina não está completamente elucidado, mas sabe-se que ela atua de forma favorável sobre a regulação do metabolismo da glicose e dos ácidos graxos, ${ }^{8}$ bem como sobre o mecanismo central da fome e do gasto energético. ${ }^{9}$

É um dos mais importantes estimuladores das vias da adenosine monophosphate-activated protein kinase (AMPK) e do peroxisome proliferator-activated receptor alpha (PPAR-alfa), além de inibir o toll-like receptor-4 (TLCR-4).$^{10}$ Inibe a atividade do fator de necrose tumoral alfa (tumor necrosis factor alpha - TNF-alfa) e, reversamente, é inibido por este fator. Além disso, é capaz de inibir outras citocinas pró-inflamatórias e de aumentar os níveis de citocinas anti-inflamatórias, como a interleucina-10 (IL-10)." Assim, atua como um potente anti-inflamatório tecidual e, em especial, parece atenuar a RI no fígado, a inflamação e a fibrose hepática. Consequentemente, seus níveis encontram-se diminuídos em pacientes com esteato-hepatite não alcoólica, quando comparados àqueles com esteatose pura. ${ }^{12}$

Encontram-se reduções dos níveis de adiponectina $^{13}$ e da sua bioatividade ${ }^{8}$ em pacientes obesos e/ou com diabetes mellitus tipo 2 (DM2), provavelmente por ação do TNF-alfa, comumente aumentado nesses pacientes. ${ }^{13}$ Há também relação inversa entre adiponectinemia e Rl, doenças cardiovasculares (DCV) e hepáticas. ${ }^{8}$ Numa série de estudos desenvolvidos no Laboratório de Pesquisas Clínicas e Experimentais de Biologia Vascular (BioVasc), da Universidade do Estado do Rio de Janeiro (UERJ), demonstrou-se que a adiponectina estava diminuída em pacientes com síndrome metabólica (SM) ${ }^{14}$ e correlacionava-se inversamente com HOMA-IR, ${ }^{15}$ gordura intramiocelular $^{15}$ (que é associada com maior Rl) e função endotelial. ${ }^{14}$ Interessantemente, após seis meses de tratamento com um agonista PPAR-gama, a rosiglitazona, os níveis da adiponectina aumentaram em mais de quatro vezes e houve redução da relação gordura intra para a extramiocelular ${ }^{15} \mathrm{e}$ normalização da vasodilatação endotélio-dependente, o que se correlacionou com o incremento da adiponectina. ${ }^{16}$

\section{Dipeptidil peptidase 4 (DPP-4)}

A DPP-4 é uma enzima ubíqua, amplamente reconhecida por clivar diversos peptídeos, entre os quais o neuropeptídeo Y (NPY), o peptídeo YY, o hormônio liberador do hormônio do crescimento (GHRH), os peptídeos semelhantes ao glucagon (GLP-1 e GLP-2) e o polipeptídeo inibitório gástrico (GIP). ${ }^{17}$ Por clivar os hormônios incretínicos, inativando-os, inibidores da DPP-4 foram desenvolvidos e são atualmente usados no tratamento do diabetes. Recentemente, Lamers e colaboradores,$^{18}$ num elegante e extenso estudo, com experimentos in vitro e clínicos com humanos, evidenciaram que a DPP-4 se expressa em, e é secretada por, adipócitos humanos diferenciados 
e macrófagos isolados do tecido adiposo. Esses autores demonstraram que a DPP-4 inibe a fosforilação da Akt in vitro e, assim, promove Rl. Além disso, observaram que seus níveis plasmáticos estão aumentados em obesos e que se correlacionam positivamente com o índice de massa corporal (IMC), insulinemia, leptinemia e todos os parâmetros da SM, enquanto correlacionam-se negativamente com a idade e a adiponectina. Adicionalmente a esse fato, se expressa mais no tecido adiposo visceral do que no subcutâneo em obesos e relaciona-se diretamente com o tamanho do adipócito. Após tratamento cirúrgico da obesidade, os níveis da DPP-4 decrescem substancialmente. Assim, estes estudos de Lamers e colaboradores definem que esta é uma nova adipocina, intrinsecamente relacionando o tecido adiposo com RI, obesidade e SM.

\section{Fator crescimento fibroblasto 21 (FGF21)}

O FGF21 (fibroblast growth factor 21) faz parte de uma grande família de fatores de crescimento polipeptídicos. É produzido e secretado predominantemente pelo fígado e em menor quantidade pelo tecido adiposo, sistema nervoso central (SNC) e pâncreas. ${ }^{19}$ Atua através de seus receptores (FGFR1, 2, 3, 4) que são tirosinoquinases acopladas a uma proteína transmembrana chamada beta-Klotho. Sua transcrição é regulada principalmente pelo PPAR-gama. ${ }^{20}$

Seus níveis plasmáticos estão aumentados em pacientes obesos, apresentando relação direta com o IMC, porém com atividade biológica reduzida. A obesidade seria um estado de resistência ao FGF21. ${ }^{21}$ No metabolismo dos carboidratos aumenta a captação de glicose pelo tecido adiposo, aumenta a sensibilidade à insulina, diminui a glucagonemia de forma dose dependente e, portanto, diminui a glicemia de jejum. Em relação aos lipídeos, promove diminuição do LDL-colesterol e dos triglicerídeos e aumento do HDL-colesterol. Aumenta o gasto energético e promove discreta perda de peso. Esses efeitos foram observados em diversos modelos animais, incluindo primatas. ${ }^{20,22}$ Embora estudos em humanos ainda sejam necessários, o FGF21 mostra-se um potencial candidato para o tratamento das alterações relacionadas à SM.

\section{Leptina}

Descoberta em 1994, a leptina é um hormônio peptídico produzido e secretado predominantemente pelo tecido adiposo, mas se expressa também em baixos níveis pelo epitélio gástrico, músculo, placenta e SNC. ${ }^{23}$

A leptina ocupa papel central na homeostase energética, bem como nas funções metabólica, reprodutiva, neuroendócrina e imunológica. No hipotálamo, atua inibindo a produção de peptídeos orexígenos como o NPY e a proteína agouti-relacionada (AgRP), bem como estimulando peptídeos anorexígenos da via pró-opiomelanocortina $(\mathrm{POMC}) /$ transcritos relacionados à anfetamina $\mathrm{e}$ à cocaína (cocaine and amphetamine regulated transcript - CART), resultando em modulação do apetite e da saciedade. ${ }^{24}$ Exerce ação sensibilizadora da insulina, mediada pelo aumento da oxidação de ácidos graxos livres e redução dos estoques de triglicerídeos no músculo esquelético, assim como regula a sua secreção através da atuação nos receptores nas células beta pancreáticas (eixo adipoinsular). Níveis de leptina estão positivamente correlacionados com os de insulina, independentemente da massa adiposa. Foram demonstradas também propriedades proliferativas e aterogênicas, associando-se ao aumento do risco cardiovascular. ${ }^{25}$

A obesidade está relacionada com aumento dos níveis séricos de leptina, numa relação direta com o IMC e a adiposidade corporal. Pacientes obesos apresentam um perfil de resistência central à ação da leptina. ${ }^{26}$ Entretanto, não se mostra eficaz no tratamento da obesidade comum, exceto nos raros casos de obesidade extrema associados à deficiência congênita da leptina. ${ }^{27} \mathrm{Em}$ outras formas de deficiência de leptina, como a observada em pacientes com lipodistrofias familiare ${ }^{28}$ ou adquiridas, ${ }^{29}$ a sua utilização também parece promissora.

\section{Omentina}

A omentina, também denominada intelectina-1, é uma adipocina identificada em 2003 a partir de um cDNA de tecido adiposo visceral omental. ${ }^{30}$ É sintetizada preferencialmente nas células do estroma vascular do tecido adiposo visceral, podendo ainda ser detectada no intesti- 
no, pulmão, coração e rins. ${ }^{31}$

Os níveis circulantes de omentina encontram-se reduzidos nos pacientes obesos ou com transtornos metabólicos associados à obesidade, como a RI e o DM2. Essa adipocina associa-se negativamente ao IMC, circunferência abdominal, leptina e HOMA-IR, e positivamente com a adiponectina e com o HDL-c. ${ }^{32}$ A administração de omentina recombinante aumenta a captação de glicose estimulada pela insulina e a fosforilação da Akt em adipócitos humanos, melhorando a sensibilidade insulínica. Especula-se que esteja envolvida na modulação do metabolismo energético e na distribuição de gordura em humanos, além de exercer ação anti-inflamatória e antiaterosclerótica. ${ }^{31}$

A omentina parece ter um efeito vasodilatador, aumentando a produção de óxido nítrico derivado do endotélio, ${ }^{33}$ apresentando relação negativa com a pressão arterial e com marcadores inflamatórios como a interleucina-6 (IL-6) e a proteína $\mathrm{C}$ reativa (PCR). Estes dados sugerem que a omentina possa representar um biomarcador não apenas para doenças metabólicas, mas também para $\mathrm{DCV}{ }^{34}$

\section{Resistina}

A resistina pertence à família RELM (Resistin -Like Molecules) de proteínas ricas em cistina. Ela foi primeiramente descrita em roedores, apresentando associação direta com obesidade e diabetes. Seus mecanismos de ação ainda não são totalmente elucidados, embora se saiba que ela atue no coração, no fígado e nos tecidos muscular e adiposo. ${ }^{35}$

Em seres humanos, a resistina se expressa em macrófagos ${ }^{36}$ e seu papel no desenvolvimento da RI é mais incerto. ${ }^{37}$ Mais evidente do que sua atuação no perfil metabólico, é o seu papel nos processos inflamatórios. A resistina tem a sua expressão estimulada por citocinas pró-inflamatórias como o TNF-alfa e a IL-6, ${ }^{38}$ e é capaz de induzir a expressão dessas citocinas no tecido adiposo e nas células mononucleares do sangue periférico. ${ }^{39}$ Assim, especula-se que essa adipocina possa representar o elo entre inflamação e sinalização metabólica.

\section{Vaspina}

A vaspina, originalmente denominada "ini- bidor da serinoprotease derivada do tecido adiposo visceral", é uma adipocina com atividade antiprotease, inicialmente isolada dos adipócitos de um modelo experimental de roedores obesos com DM2, conhecidos por Otsuka Long-Evans Tokushima Fatty (OLETF). Nesses animais, foi demonstrado que a maior expressão do RNAm da vaspina no tecido adiposo visceral coincide com o pico da obesidade e RI. ${ }^{40}$ Em humanos, a vaspina foi inicialmente identificada no tecido adiposo, embora esteja presente também no estômago, fígado, pâncreas, hipotálamo e pele. ${ }^{41}$

Embora os mecanismos de ação da vaspina ainda não estejam completamente elucidados, postula-se que essa adipocina poderia representar um mecanismo compensatório associado à obesidade e à RI grave. ${ }^{40,41} \mathrm{~A}$ administração de vaspina foi capaz de promover diminuição da glicemia e da ingestão alimentar de roedores, sugerindo que essa adipocina seja capaz de deflagrar vias anorexígenas no hipotálamo, possivelmente através da redução das concentrações de NPY e do aumento do RNAm da POMC. Outra hipótese seria que essa adipocina exerceria um efeito inibitório sobre uma protease capaz de degradar um fator antiorexígeno. ${ }^{42}$

\section{Visfatina}

A visfatina é uma adipocina de $52 \mathrm{kDa}$ identificada em 2004. ${ }^{43}$ A origem do seu nome tem como base a concepção de que ela seria sintetizada e secretada pela gordura visceral. ${ }^{44}$ Entretanto, reconhece-se atualmente que a visfatina é predominantemente sintetizada por leucócitos, incluindo os macrófagos que infiltram o tecido adiposo visceral, em resposta a sinais inflamatórios. ${ }^{36}$

As ações da visfatina influenciam fenômenos inflamatórios, ateroscleróticos e a secreção insulínica. ${ }^{44} \mathrm{~A}$ visfatina favorece as vias relacionadas à inflamação, ao promover o aumento da síntese de citocinas pró-inflamatórias pelos monócitos, tais como a interleucina-1b (IL-1b), a IL-6 e o TNF -alfa.$^{45}$

A patogênese da doença macrovascular parece ser influenciada pela visfatina, uma vez que a expressão crônica dessa adipocina apresenta efeito aterogênico. Ela é abundante nas placas aterogênicas obtidas de pacientes com infarto agudo do miocárdio e é capaz de ativar a expres- 
são de matriz metaloproteinase $2 / 9$ em células endoteliais humanas, que está implicada na via de instabilidade da placa. ${ }^{46}$

A visfatina está significativamente aumentada em indivíduos com excesso de peso, DM2, SM e DCV, bem como possivelmente relacionada à RI. ${ }^{46}$ Em pacientes com DM2, os níveis de visfatina estão associados a maiores valores de hemoglobina glicada. ${ }^{47}$

Apesar dessas associações, há evidências experimentais de que a visfatina apresenta efeitos insulinomiméticos e de que é capaz de regular a secreção de insulina, a fosforilação do receptor insulínico e a expressão de genes nas células beta de ratos. ${ }^{43}$

Contrariando os efeitos relacionados à exposição crônica à visfatina, a exposição aguda a essa adipocina parece trazer benefícios em situações especiais. Evidenciou-se um efeito cardioprotetor em modelo animal de isquemia não aterosclerótica quando a visfatina foi administrada no momento da reperfusão, com redução do tamanho da área infartada. ${ }^{48}$

\section{Conclusão}

As adipocinas compõem um grupo de substâncias que se expressam e são secretadas pelo tecido adiposo. Embora o papel da maioria delas ainda não esteja claramente definido, pode-se afirmar que são intrinsecamente relacionadas com estados de excesso e distribuição da gordura corporal. A utilidade destas substâncias na prática clínica, portanto, passa desde marcadores positivos de excesso de peso e Rl, como a leptina, a DPP-4, a vaspina e a visfatina, e negativos como a adiponectina, o FGF-21, a indutores de inflamação e aterogênese. Destas, apenas a DPP-4 (recentemente caracterizada como uma adipocina) e a leptina já se apresentam como potenciais alvos ou agentes terapêuticos.

\section{Referências}

1. Gastelurrutia P, Pascual-Figal D, Vazquez R, Cygankiewicz I, Shamagian LG, Puig T, et al. Obesity paradox and risk of sudden death in heart failure results from the MUerte Subita en Insuficiencia Cardiaca (MUSIC) study. Am Heart J. 2011 Jan;161(1):158-64. http://dx.doi. org/10.1016/j.ahj.2010.10.018

2. Ouchi N, Parker JL, Lugus JJ, Walsh K. Adipokines in inflammation and metabolic disease. Nat Rev Immunol. 2011 Feb;11(2):85-97. http://dx.doi.org/10.1038/ nri2921

3. Trayhurn P, Wood IS. Adipokines: inflammation and the pleiotropic role of white adipose tissue. $\mathrm{Br}$ J Nutr. 2004 Sep;92(3):347-55.

4. Fain JN, Madan AK, Hiler ML, Cheema P, Bahouth SW. Comparison of the release of adipokines by adipose tissue, adipose tissue matrix, and adipocytes from visceral and subcutaneous abdominal adipose tissues of obese humans. Endocrinology. 2004 May;145(5):2273-82.

5. Scherer PE, Williams S, Fogliano M, Baldini G, Lodish HF. A novel serum protein similar to C1q, produced exclusively in adipocytes. J Biol Chem. $1995 \mathrm{Nov}$ 10;270(45):26746-9.

6. Doran AC, Meller N, Cutchins A, Deliri H, Slayton RP, Oldham SN, et al. The helix-loop-helix factors Id3 and E47 are novel regulators of adiponectin. Circ Res. 2008 Sep 12;103(6):624-34. http://dx.doi. org/10.1161/CIRCRESAHA.108.175893

7. Wang Y, Xu A, Knight C, Xu LY, Cooper GJ. Hydroxylation and glycosylation of the four conserved lysine residues in the collagenous domain of adiponectin. Potential role in the modulation of its insulin-sensitizing activity. J Biol Chem. 2002 May 31;277(22):19521-9.

8. Shehzad A, Iqbal W, Shehzad O, Lee YS. Adiponectin: regulation of its production and its role in human diseases. Hormones (Athens). 2012 Jan-Mar;11(1):8-20.

9. Kubota N, Yano W, Kubota T, Yamauchi T, Itoh S, Kumagai $\mathrm{H}$, et al. Adiponectin stimulates AMP-activated protein kinase in the hypothalamus and increases food intake. Cell Metab. 2007 Jul;6(1):55-68.

10. Kadowaki T, Yamauchi T, Kubota N, Hara K, Ueki K, Tobe K. Adiponectin and adiponectin receptors in insulin resistance, diabetes, and the metabolic syndrome. J Clin Invest. 2006 Jul;116(7):1784-92.

11. Polyzos SA, Kountouras J, Zavos C, Tsiaousi E. The role of adiponectin in the pathogenesis and treatment of non-alcoholic fatty liver disease. Diabetes Obes Metab. 2010 May;12(5):365-83. http://dx.doi. org/10.1111/j.1463-1326.2009.01176.x

12. Younossi ZM, Jarrar M, Nugent C, Randhawa M, Afendy M, Stepanova M, et al. A novel diagnostic biomarker panel for obesity-related nonalcoholic steatohepatitis (NASH). Obes Surg. 2008 Nov;18(11):14307. http://dx.doi.org/10.1007/s11695-008-9506-y

13. Ouchi N, Kihara S, Funahashi T, Matsuzawa Y, Walsh K. Obesity, adiponectin and vascular inflammatory disease. Curr Opin Lipidol. 2003 Dec;14(6):561-6.

14. Bahia L, Aguiar LG, Villela N, Bottino D, Godoy-Matos AF, Geloneze B, et al. Relationship between adipokines, inflammation, and vascular reactivity in 
lean controls and obese subjects with metabolic syndrome. Clinics (Sao Paulo). 2006 Oct;61(5):433-40.

15. Godoy-Matos AF, Bahia LR, Domingues RC, Sicuro F, Tambascia M, Geloneze B, et al. Adiponectin is related to intramyocellular lipid content in non-diabetic adults. J Endocrinol Invest. 2010;33(6):382-7. http:// dx.doi.org/10.3275/6474

16. Bahia L, Aguiar LG, Villela N, Bottino D, Godoy-Matos AF, Geloneze B, et al. Adiponectin is associated with improvement of endothelial function after rosiglitazone treatment in non-diabetic individuals with metabolic syndrome. Atherosclerosis. 2007 Nov;195(1):138-46.

17. Mentlein R. Dipeptidyl-peptidase IV (CD26)--role in the inactivation of regulatory peptides. Regul Pept. 1999 Nov 30;85(1):9-24.

18. Lamers D, Famulla S, Wronkowitz N, Hartwig S, Lehr S, Ouwens DM, et al. Dipeptidyl peptidase 4 is a novel adipokine potentially linking obesity to the metabolic syndrome. Diabetes. 2011 Jul;60(7):1917-25. http://dx.doi.org/10.2337/db10-1707

19. Ornitz DM, Itoh N. Fibroblast growth factors. Genome Biol. 2001;2(3):REVIEWS3005.

20. Kliewer SA, Mangelsdorf DJ. Fibroblast growth factor 21: from pharmacology to physiology. Am J Clin Nutr. 2010 Jan;91(1):254S-7S. http://dx.doi.org/10.3945/ ajcn.2009.28449B

21. Fisher FM, Chui PC, Antonellis PJ, Bina HA, Kharitonenkov A, Flier JS, et al. Obesity is a fibroblast growth factor 21 (FGF21)-resistant state. Diabetes. 2010 Nov;59(11):2781-9. http://dx.doi.org/10.2337/ db10-0193

22. Xu J, Lloyd DJ, Hale C, Stanislaus S, Chen M, Sivits $\mathrm{G}$, et al. Fibroblast growth factor 21 reverses hepatic steatosis, increases energy expenditure, and improves insulin sensitivity in diet-induced obese mice. Diabetes. 2009 Jan;58(1):250-9. http://dx.doi. org/10.2337/db08-0392

23. Masuzaki H, Ogawa Y, Sagawa N, Hosoda K, Matsumoto $\mathrm{T}$, Mise $\mathrm{H}$, et al. Nonadipose tissue production of leptin: leptin as a novel placenta-derived hormone in humans. Nat Med. 1997 Sep;3(9):1029-33.

24. Sahu A. Leptin signaling in the hypothalamus: emphasis on energy homeostasis and leptin resistance. Front Neuroendocrinol. 2003 Dec;24(4):225-53.

25. Ahima RS, Flier JS. Leptin. Annu Rev Physiol. 2000;62:413-37.

26. Kim JY, van de Wall E, Laplante M, Azzara A, Trujillo ME, Hofmann SM, et al. Obesity-associated improvements in metabolic profile through expansion of adipose tissue. J Clin Invest. 2007 Sep;117(9):2621-37.

27. Farooqi IS, Matarese G, Lord GM, Keogh JM, Lawrence $\mathrm{E}$, Agwu $\mathrm{C}$, et al. Beneficial effects of leptin on obesity, T cell hyporesponsiveness, and neuroendocrine/metabolic dysfunction of human congenital leptin deficiency. J Clin Invest. 2002 Oct;110(8):1093103.

28. Mantzoros CS. Leptin in relation to the lipodystrophy-associated metabolic syndrome. Diabetes Metab J. 2012 Jun;36(3):181-9. http://dx.doi.org/10.4093/ dmj.2012.36.3.181

29. Magkos F, Brennan A, Sweeney L, Kang ES, Doweiko J, Karchmer AW, et al. Leptin replacement improves postprandial glycemia and insulin sensitivity in human immunodeficiency virus-infected lipoatrophic men treated with pioglitazone: a pilot study. Metabolism. 2011 Jul;60(7):1045-9. http://dx.doi.org/10.1016/j. metabol.2010.10.002

30. Yang R, Xu A, Pray J, Hu H, Jadhao S, Hansen B, et al. Cloning of omentin, a new adipocytokine from omental fat tissue in humans. Diabetes. 2003;Suppl 1:A1.

31. Yang RZ, Lee MJ, Hu H, Pray J, Wu HB, Hansen BC, et al. Identification of omentin as a novel depot-specific adipokine in human adipose tissue: possible role in modulating insulin action. Am J Physiol Endocrinol Metab. 2006 Jun;290(6):E1253-61.

32. Tan BK, Adya R, Farhatullah S, Lewandowski KC, O'Hare P, Lehnert H, et al. Omentin-1, a novel adipokine, is decreased in overweight insulin-resistant women with polycystic ovary syndrome: ex vivo and in vivo regulation of omentin-1 by insulin and glucose. Diabetes. 2008 Apr;57(4):801-8. http://dx.doi. org/10.2337/db07-0990

33. Yamawaki H, Tsubaki N, Mukohda M, Okada M, Hara Y. Omentin, a novel adipokine, induces vasodilation in rat isolated blood vessels. Biochem Biophys Res Commun. 2010 Mar 19;393(4):668-72. http://dx.doi. org/10.1016/j.bbrc.2010.02.053

34. Shibata R, Ouchi N, Takahashi R, Terakura Y, Ohashi $\mathrm{K}$, lkeda $\mathrm{N}$, et al. Omentin as a novel biomarker of metabolic risk factors. Diabetol Metab Syndr. 2012 Jul 26;4(1):37. http://dx.doi.org/10.1186/1758-5996-437

35. Steppan CM, Brown EJ, Wright CM, Bhat S, Banerjee $\mathrm{RR}$, Dai CY, et al. A family of tissue-specific resistin-like molecules. Proc Natl Acad Sci U S A. 2001 Jan 16;98(2):502-6.

36. Curat CA, Wegner V, Sengenès C, Miranville A, Tonus $\mathrm{C}$, Busse R, et al. Macrophages in human visceral adipose tissue: increased accumulation in obesity and a source of resistin and visfatin. Diabetologia. 2006 Apr;49(4):744-7.

37. Heilbronn LK, Rood J, Janderova L, Albu JB, Kelley DE, Ravussin E, et al. Relationship between serum resistin concentrations and insulin resistance in nonobese, obese, and obese diabetic subjects. J Clin Endocrinol Metab. 2004 Apr;89(4):1844-8. 
38. Kaser S, Kaser A, Sandhofer A, Ebenbichler CF, Tilg H, Patsch JR. Resistin messenger-RNA expression is increased by proinflammatory cytokines in vitro. Biochem Biophys Res Commun. 2003 Sep 19;309(2):286-90.

39. Bokarewa M, Nagaev I, Dahlberg L, Smith U, Tarkowski A. Resistin, an adipokine with potent proinflammatory properties. J Immunol. 2005 May 1;174(9):5789-95.

40. Li Q, Chen R, Moriya J, Yamakawa J, Sumino H, Kanda T, et al. A novel adipocytokine, visceral adipose tissue-derived serine protease inhibitor (vaspin), and obesity. J Int Med Res. 2008 Jul-Aug;36(4):625-9.

41. Blüher M. Vaspin in obesity and diabetes: pathophysiological and clinical significance. Endocrine. 2012 Apr;41(2):176-82. http://dx.doi.org/10.1007/s12020011-9572-0

42. Klöting N, Kovacs P, Kern M, Heiker JT, Fasshauer $\mathrm{M}$, Schön MR, et al. Central vaspin administration acutely reduces food intake and has sustained blood glucose-lowering effects. Diabetologia. 2011 Jul;54(7):1819-23. http://dx.doi.org/10.1007/s00125011-2137-1

43. Fukuhara A, Matsuda M, Nishizawa M, Segawa K, Tanaka M, Kishimoto K, et al. Visfatin: a protein secreted by visceral fat that mimics the effects of insulin. Science. 2005 Jan 21;307(5708):426-30.

44. Saddi-Rosa P, Oliveira CS, Giuffrida FM, Reis AF. Visfatin, glucose metabolism and vascular disease: a review of evidence. Diabetol Metab Syndr. 2010 Mar 26;2:21. http://dx.doi.org/10.1186/1758-5996-2-21

45. Tilg H, Moschen AR. Role of adiponectin and PBEF/ visfatin as regulators of inflammation: involvement in obesity-associated diseases. Clin Sci (Lond). 2008 Feb;114(4):275-88. http://dx.doi.org/10.1042/ CS20070196

46. Chang YH, Chang DM, Lin KC, Shin SJ, Lee YJ. Visfatin in overweight/obesity, type 2 diabetes mellitus, insulin resistance, metabolic syndrome and cardiovascular diseases: a meta-analysis and systemic review. Diabetes Metab Res Rev. 2011 Sep;27(6):51527. http://dx.doi.org/10.1002/dmrr.1201

47. López-Bermejo A, Chico-Julià B, Fernàndez-Balsells M, Recasens M, Esteve E, Casamitjana R, et al. Serum visfatin increases with progressive beta-cell deterioration. Diabetes. 2006 Oct;55(10):2871-5.

48. Lim SY, Davidson SM, Paramanathan AJ, Smith CC, Yellon DM, Hausenloy DJ. The novel adipocytokine visfatin exerts direct cardioprotective effects. J Cell Mol Med. 2008 Aug;12(4):1395-403. http://dx.doi. org/10.1111/j.1582-4934.2008.00332.x.

\footnotetext{
${ }^{* *}$ Brazilian Adipokines Study Group:
}

Amélio F. Godoy-Matos, Alessandra Schiappacassa, Aline A. Lacerda, Amanda D. D. Bicudo, Ana Luiza Soldera, Anna Carolina A. Guimarães, Bruno Cury, Carolina G. Meireles, Ciciliana M. Z. Rech, Daniela T. Mendes, Elisiane R. Baldissera, Ivan C. Cruz, Jordana C. Rodrigues, Juliana C. Rodrigues, Juliana S. Paula, Juliana J. Laibida, Juliana S. Almeida, Letícia D. C. Braga, Luisa B. P. Soares, Mariana P. F. A. R. Cunha, Priscila O. Siciliano, Rafael da Costa, Wellington S. Silva Júnior.

Recebido: $\quad$ 19/08/2013.

Revisado: $\quad$ 26/11/2013.

Aprovado: $\quad$ 04/02/2014. 